\title{
Buffer capacity of the coelomic fluid in echinoderms
}

\author{
Laitat K., ", M. Collard ${ }^{1,2, *,+}$, L. Moulin", ${ }^{1,3}$, A.I. Catarino', Ph. Grosjean ${ }^{3}$ and Ph. Dubois ${ }^{1}$ \\ 1 Laboratoire de Biologie Marine, Université Libre de Bruxelles, 50 avenue F.D. Roosevelt, B-1050 \\ Brussels, Belgium \\ E-mail: marie.collard9@gmail.com \\ 2 Laboratorium voor Analytische Chemie, Vrije Universiteit Brussel, Pleinlaan 2, B-1 050 Brussels, \\ Belgium \\ 3 Laboratoire d'Ecologie Numérique des Milieux Aquatiques, Université de Mons-Hainaut, 6 Avenue \\ du Champ de Mars, B-7000 Mons, Belgium \\ * These authors contributed equally to this work.
}

The increase in atmospheric $\mathrm{CO}_{2}$ due to anthropic activities results in an acidification of the surface waters of the oceans. The impact of these chemical changes differs according to the considered organisms. The intertidal rocky shores may harbor organisms pre-adapted to the upcoming changes as they already face tidal $\mathrm{pH}$ and temperature fluctuations. In order to cope with the changes in seawater $\mathrm{pH}$, these organisms possess different mechanisms involved in acid-base regulation. Some organisms present a higher buffer capacity than seawater, among which echinoderms. The properties of this buffer capacity and the factors influencing it were investigated in the sea urchin Paracentrotus lividus and in the starfish Asterias rubens, both species occurring in the intertidal zone of the North Atlantic and the North Sea, respectively. Buffer capacity is partly due to the coelomocytes present in the coelomic fluid and, in P. lividus, it is also due to a compound which contributes to a higher buffer capacity of the coelomic fluid of this species compared to that of the starfish. The effect of a decreased seawater $\mathrm{pH}$ (in the scope of predicted future ocean acidification) on this buffer capacity in $P$. lividus was investigated. A gradual increase of the buffer capacity was recorded when the seawater $\mathrm{pH}$ was decreased. Moreover, the comparison of different echinoderm species showed that Euechinoidea present a very high buffer capacity while Cidadroidea (other sea urchins), starfish and holothurians have a lower one. This can be explained either by the presence of the compound only in Euechinoidea, linked to differences in the respiratory machinery, or by metabolic differences between the various classes of echinoderms.

Keywords: ocean acidification; echinoderms; acid-base regulation. 